\title{
Production of the new cholera toxin by environmental isolates of Vibrio cholerae non-01
}

\author{
D. V. SINGH, ANJALI TIKOO and S. C. SANYAL* \\ Department of Microbiology, Institute of Medical Sciences, Banaras Hindu University, Varanasi 221 005, India
}

\begin{abstract}
One of five strains of Vibrio cholerae non-O1 isolated from environmental sources caused fluid accumulation in an initial rabbit ileal loop (RIL) test. The four strains that caused little or no accumulation of fluid gave a positive response after one-to-three consecutive passages through RILs. The amount of fluid produced increased after each passage. Filtrates of cultures of all five environmental isolates caused fluid accumulation similar to that produced by live cells. The enterotoxin showed a precipitin band with new cholera antitoxin and was neutralised completely by new cholera antitoxin diluted 1 in 32, indicating its close immunobiological relationship to the new cholera toxin. The present study indicates that $V$. cholerae non-O1 strains produce an enterotoxin that is similar to the new cholera toxin.
\end{abstract}

\section{Introduction}

Vibrio cholerae non-O1 has been recognised as a causative agent of sporadic and localised outbreaks of cholera-like diarrhoea [1]. This organism has also been implicated as a cause of extra-intestinal infections such as wound infection, septicaemia and cellulitis in man [2-4]. A large number of $V$. cholerae non-Ol strains of clinical and environmental origins produce an enterotoxin that is similar immunologically and biologically to cholera toxin (CT) [5-10]. Recently, several other extracellular products, all of which may play an important role in the pathogenesis of the disease, have been documented. These include heat-stable enterotoxin (NAG-ST) similar to the heat-stable enterotoxin of Escherichia coli that caused fluid accumulation in an infant mouse model [11-13], a thermostable direct haemolysin similar to that of $V$. parahaemolyticus $[12,14]$, a heat-labile El Tor-like haemolysin [15-17], a shiga-like toxin [18], haemagglutinins $[19,20]$ and Zonula Occludens Toxin (Zot) [7]. It seems that the virulence of $V$. cholerae non-O1 is multifactorial and is mediated by several traits in an integrated fashion [7]. However, there is no published information about the production of new cholera toxin (NCT) by $V$. cholerae non-O1. NCT can be produced by cholera toxin genenegative and -positive strains of $V$. cholerae $\mathrm{O} 1$, both classical and El Tor biotypes from both clinical and environmental origins [21-26]. Therefore, the present study of environmental isolates of $V$. cholerae non-O1 was undertaken to see whether fluid accumulation in

Received 1 Aug. 1995; accepted 17 Nov. 1995.

* Corresponding author: Professor S. C. Sanyal.
RILs following inoculation with culture filtrates could be neutralised by an antitoxin against NCT produced by CT gene-negative $V$. cholerae $\mathrm{O} 1$. The study also investigated whether there is any antigenic similarity with NCT produced by a CT gene-negative $V$. cholerae $\mathrm{O} 1$ strain and the toxin(s) produced by non-O1 environmental isolates.

\section{Materials and methods}

\section{Bacterial strains}

Five strains of $V$. cholerae non-O1 isolated from the environment by this laboratory were identified by standard techniques [27]. A CT gene-negative $V$. cholerae O1 (strain X-392) which produced NCT was obtained from J. B. Kaper, Center for Vaccine Development, MD, USA, and included for preparation of antiserum against purified NCT.

\section{Medium}

Preliminary tests for toxin production were made in AKI medium. The AKI medium (Bacto-peptone 1.5\% $\mathrm{v} / \mathrm{v}$, yeast extract $0.4 \%, \mathrm{NaCl} 0.5 \%$ and $\mathrm{NaHCO}_{3} 0.3 \%$ ) was prepared as described by Iwanaga et al. [28]. The sodium bicarbonate was filter sterilised separately and mixed with the other autoclaved ingredients. Freshly prepared medium was always used; the $\mathrm{pH}$ of this medium was $c .7 .4$ without adjustment.

\section{Preparation of culture filtrates}

Culture filtrates (CFs) of the five isolates of $V$. cholerae non-O1, once their live cells were known to cause fluid 
accumulation in ileal loops, were prepared by the method of Annapurna and Saynal [29]. Briefly, $10 \mathrm{ml}$ of AKI medium in a $50-\mathrm{ml}$ conical flask were inoculated with five or six colonies from an overnight nutrient agar plate culture. The flasks were incubated at $37^{\circ} \mathrm{C}$ in a water-bath with shaking for $16-18 \mathrm{~h}$ with 80 120 oscillations/min. The cultures were centrifuged at $22000 \mathrm{~g}$ for $20 \mathrm{~min}$ at $4^{\circ} \mathrm{C}$ and supernates were filtered through membrane filters (Millipore, $0.22 \mu \mathrm{m}$ ) and stored in small volumes at $4^{\circ} \mathrm{C}$.

\section{Ileal loop test}

Live cells of the five $V$. cholerae non-O1 isolates were tested for enterotoxin production in adult New Zealand and albino rabbits by the method of De and Chatterje [30]. The strains were grown in peptone water for $3-4 \mathrm{~h}$, diluted 10 -fold in the same medium and inoculated in RILs in doses of $1 \mathrm{ml}\left(10^{5-6} \mathrm{cfu}\right)$. The known toxigenic $V$. cholerae strain $956 \mathrm{~B}$ grown in peptone water and unseeded peptone water served as positive and negative controls, respectively. CFs of all these strains prepared in AKI medium were tested in a similar way with 1-ml inocula. Each test was done in duplicate.

\section{Passage through RILs}

Consecutive passages of the $V$. cholerae non-O1 strains that caused little or no accumulation of fluid in the initial test were made in RILs as described earlier [6,22]. Briefly, such strains were recovered from the ileal loops on nutrient agar plates and, following overnight incubation, five or six colonies were inoculated into peptone water, incubated at $37^{\circ} \mathrm{C}$ for $3-4 \mathrm{~h}$ and $1 \mathrm{ml}$ of diluted culture was inoculated again into RIL. This process was continued until good positive responses were obtained.

\section{Determination of the optimum loop reacting dose}

Cell-free CFs of five strains of $V$. cholerae non-Ol were injected in volumes ranging between 0.05 and $1.0 \mathrm{ml}$ into RILs to find the dose causing a maximal reaction [29].

\section{Neutralisation of $V$. cholerae non-Ol enterotoxin by NCT in RIL}

CFs of five strains in $0.15-\mathrm{ml}$ amounts contained in $0.5 \mathrm{ml}$ of phosphate-buffered saline (PBS, $\mathrm{pH} 7.4$ ) were mixed with equal volumes of anti-NCT serum in serial doubling dilutions as described previously [6, 22, 31]. The mixtures were incubated at $37^{\circ} \mathrm{C}$ in a water-bath for $30 \mathrm{~min}$ and then tested in RILs to find the highest dilution of anti-NCT serum that would completely neutralise the enterotoxic activity in CFs. CF-PBS mixture and PBS alone served as positive and negative controls, respectively.

\section{Immunodiffusion test}

Ouchterlony immunodiffusion tests were performed with 10-fold concentrated CFs (with polyethylene glycol 4000, Ranbaxy, India) of the test strains against anti-NCT serum. CF of strain X-392 along with those of $V$. cholerae non-O1 strains were tested individually against anti-NCT serum.

\section{Results}

In the initial experiments, live cells of only one of the five isolates tested caused fluid accumulation in RILs, comparable to that of $V$. cholerae $\mathrm{O} 1$ toxigenic strain $569 \mathrm{~B}$. Of the four isolates that caused little or no accumulation of fluid in the initial tests, two each became positive after two and three passages through rabbit gut, respectively (Table 1). These strains showed marked increase in fluid accumulation on each consecutive passage through the rabbit gut (data not shown). CFs of all these strains also caused fluid accumulation in the range similar to those produced by live cells.

Filtrates in doses of $0.15 \mathrm{ml}$ caused fluid accumulations in the range $0.74-1.2 \mathrm{ml} / \mathrm{cm}$ of RIL. The amount of fluid accumulation was no greater when larger volumes of CFs were injected (Fig. 1). The 0.15-ml dose of CF was used in neutralisation tests with the antiserum against NCT.

The highest dilution of anti-NCT serum that completely neutralised the enterotoxic activity in these CFs was 1 in 32. There was proportionately less neutralisation, i.e., more fluid accumulation, with higher dilutions of anti-NCT serum.

When CFs of the CT gene-negative $V$. cholerae $\mathrm{O} 1$ (strain X-392) and $V$. cholerae non-O1 strains were tested in gel diffusion, one (88-SH) gave a precipitin band against the NCT antiserum showing reaction of identity, whereas the remaining four showed a reaction of partial identity.

Table 1. Enhancement of fluid accumulation of $V$. cholerae non-O1 strains after passage through RILs

\begin{tabular}{|c|c|c|}
\hline \multirow[b]{2}{*}{ Strain no. } & \multicolumn{2}{|c|}{$\begin{array}{c}\text { Fluid accumulation } \\
\text { ( } \mathrm{ml} / \mathrm{cm} \text { of rabbit ileal loop) }\end{array}$} \\
\hline & Before passage & $\begin{array}{c}\text { After passage } \\
\text { (number of passages) }\end{array}$ \\
\hline 86-HG & 0 & $0.5-0.9(3)$ \\
\hline 86-TG-2 & 0 & $0.7-1.1$ \\
\hline 88-AS & 0 & $0.6-0.7(2)$ \\
\hline 88-SH & 0 & $0.5-0.7(2)$ \\
\hline 86-DSM & $0.5-0.9$ & $0.7-1.2(0)$ \\
\hline Positive control ${ }^{*}$ & $0.8-1.1$ & $1.0-1.4$ \\
\hline Negative control $^{\dagger}$ & 0 & 0 \\
\hline
\end{tabular}

*Peptone water culture of $V$. cholerae strain 569B.

${ }^{\dagger}$ Peptone water. 


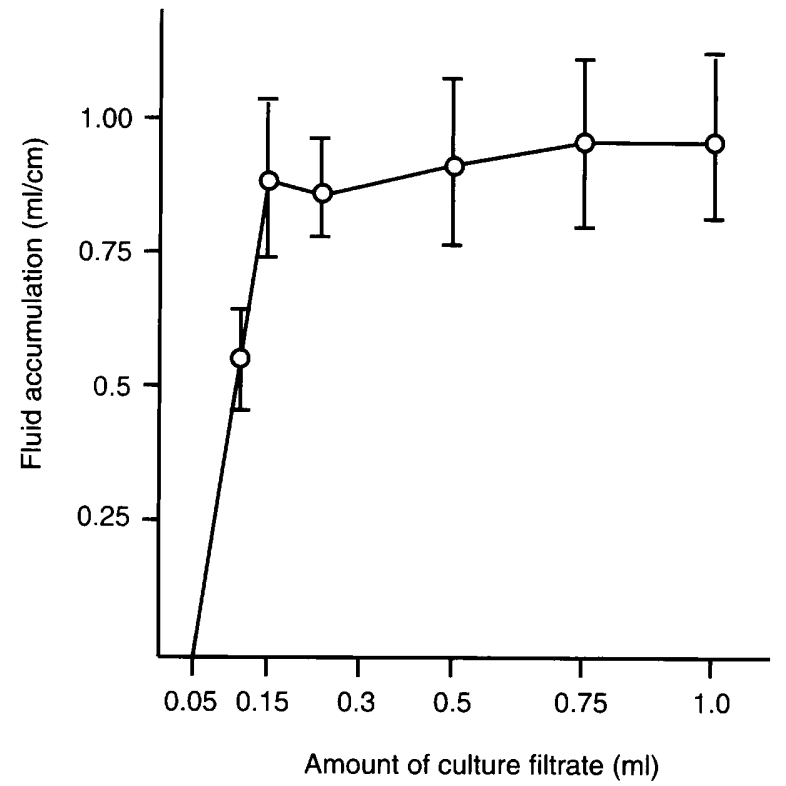

Fig. 1. Determination of optimal loop reacting dose accumulation of fluid with different doses of CFs. The results are means $(\mathrm{bar}=\mathrm{SD})$ for filtrates of five cultures of $V$. cholerae non-O1.

\section{Discussion}

The present study suggests that all strains of $V$. cholerae non-O1 produce enterotoxin as judged by fluid accumulation in RILs, the classical model for detection of enterotoxin activity of bacteria. Moreover, strain-to-strain and loop-to-loop variations in fluid accumulation as observed might have been due to variations in the amounts of toxin released by them and to biological variations [32].

Four of the five isolates of $V$. cholerae non-O1 that caused little or no accumulation of fluid in the initial test showed enhancement of enterotoxic activity after one-to-three consecutive passages through the gut of a susceptible host, i.e., rabbit ileum. Fluid outpouring increased after each passage. Similar effects of passage on enterotoxigenicity have been observed earlier with $V$. cholerae non-O1 $[32,33]$, and with other organisms such as $V$. cholerae $\mathrm{O} 1$ [21,22], Aeromonas spp. [34-37], Plesiomonas shigelloides [38], V. fluvialis [39], V. mimicus [40] and Klebsiella pneumoniae [41]. Furthermore, it is well known that the so-called hypertoxigenic strain $569 \mathrm{~B}$ of $V$. cholerae $\mathrm{O} 1$ also requires passage through rabbit gut for enhancement of toxin production. A mechanism of repression of a toxin gene that becomes expressed on passage in a susceptible host may account for this phenomenon [32]. There is also a possibility that toxigenicity of the isolates might have decreased during subculture or storage in the laboratory before the ileal loop tests. It appears that repressionderepression of a virulence factor in a bacterial strain is a means for its adaptation to a particular microenvironment. The present study thus suggests that all the $V$. cholerae non-O1 strains were potentially enterotoxigenic.

The observation that the toxin produced by one $V$. cholerae non-O1 strain (no. 88 -SH) gave a precipitin band against anti-NCT serum showing reaction of identity suggests that this strain produced an enterotoxin antigenically similar to NCT. This conclusion is further reinforced by the fact that the enterotoxic activity of this strain was completely neutralised by anti-NCT serum at a dilution of 1 in 32 .

The CFs of the four other strains showed only partial identity with NCT and complete neutralisation of enterotoxic activity in ileal loops, suggesting that these strains also produced NCT but possibly with some weaker epitopes, as has been observed in CT-B subunits produced by classical and El Tor biotype strains [42]. Tamplin et al. [43] also observed five shared and one unshared epitope between classical and El Tor CFs as well as some variation in the extent of cross-reactivity between different El Tor CT-B preparations. Although the subunit structure of NCT has not yet been determined, its large mol. wt (61000, unpublished data) suggests that this toxin possesses some subunits, the epitopes of which may differ slightly from strain to strain. However, these differences are minor and do not affect the neutralising capability of the antitoxin prepared against strain X392.

This study thus suggests that the strains of $V$. cholerae non-O1 isolated from environmental sources produce an enterotoxin that is similar to the new cholera toxin.

The study was supported in part by the Council of Scientific and Industrial Research, New Delhi in the form of a Research Associateship to D.V.S. and Senior Research Fellowship to A. T. The authors thank Mr D. Venu Gopal for secretarial assistance.

\section{References}

1. Blake PA, Weaver RW, Hollis DG. Diseases of humans (other than cholera) caused by vibrios. Annu Rev Microbiol 1980; 34: 341-367.

2. Gelbart SM, Praphudesai MM. Vibrio cholerae non-O1 cellulitis. Arch Pathol Lab Med 1986; 110: 1182-1183.

3. Hughes JM, Hollis DG, Gangarosa EJ, Weaver RE. Noncholera vibrio infections in the United States. Clinical, epidemiologic, and laboratory features. Ann Intern Med 1978; 88: 602-606.

4. Safrin S, Morris JG, Adams M, Pons V, Jacob R, Conte JE. Non-O:1 Vibrio cholerae bacteremia: case report and review. Rev Infect Dis 1988; 10: 1012-1017.

5. Craig JP, Yamamoto K, Takeda Y, Miwatani T. Production of cholera-like enterotoxin by a Vibrio cholerae non-O1 strain isolated from the environment. Infect Immun 1981; 34: 90-97.

6. Lahiri A, Agarwal RK, Sanyal SC. Biological similarity of enterotoxins of Vibrio cholerae serotypes other than type 1 to cholera toxin and Escherichia coli heat-labile enterotoxin. $J$ Med Microbiol 1982; 15: 429-440.

7. Ramamurthy T, Bag PK, Pal A et al. Virulence patterns of Vibrio cholerae non-O1 strains isolated from hospitalised patients with acute diarrhoea in Calcutta, India. $J \mathrm{Med}$ Microbiol 1993; 39: 310-317. 
8. Sanyal SC. NAG Vibrio toxin. In: Dorner F, Drews J (eds) Pharmacology of bacterial toxins. International encyclopedia of pharmacology and therapeutics, 119. Oxford, Pergamon Press. 1986: 207-225.

9. Yamamoto K, Takeda Y, Miwatani T, Craig JP. Evidence that a non-O1 Vibrio cholerae produces enterotoxin that is similar but not identical to cholera enterotoxin. Infect Immun 1983; 41: 896-901.

10. Zinaka Y, Carpenter CC. An enterotoxin produced by noncholera vibrios. Johns Hopkins Med J 1972; 131: 403-411.

11. Arita M, Takeda $T$, Honda $T$, Miwatani $T$. Purification and characterization of Vibrio cholerae non-O1 heat-stable enterotoxin. Infect Immun 1986; 52: 45-49.

12. Honda $T$, Arita $M$, Takeda $T$, Yoh $M$, Miwatani $T$. Non-O Vibrio cholerae produces two newly identified toxins related to Vibrio parahaemolyticus haemolysin and Escherichia coli heatstable enterotoxin. Lancet $1985 ; 2$ : 163-164.

13. Takao $T$, Shimonishi $Y$, Kobayashi $M$ et al. Amino acid sequence of heat-stable enterotoxin produced by Vibrio cholerae non-O1. FEBS Lett 1985; 193: 250-254.

14. Yoh M, Honda T, Miwatani T. Production by non $\mathrm{O} 1$ Vibrio cholerae haemolysin related to thermostable direct haemolysin of Vibrio parahemolyticus. FEMS Microbiol Lett 1985; 29: 197-200.

15. Ichinose $\mathrm{Y}$, Yamamoto $\mathrm{K}$, Nakasone $\mathrm{N}$ et al. Enterotoxicity of El Tor-like hemolysin of non-O1 Vibrio cholerae. Infect Immun 1987; 55: 1090-1093.

16. Yamamoto K, Al-Omani M, Honda T, Takeda Y, Miwatani T. Non-O1 Vibrio cholerae hemolysin: purification, partial characterization, and immunological relatedness to El Tor hemolysin. Infect Immun 1984; 45: 192-196.

17. Yamamoto $\mathrm{K}$, Ichinose $\mathrm{Y}$, Nakasone $\mathrm{N}$ et al. Identity of hemolysins produced by Vibrio cholerae non-O1, and $V$. cholerae O1, biotype El Tor. Infect Immun 1986; 51: 927-931.

18. O'Brien AD, Chen ME, Holmes RK, Kaper JB, Levine MM. Environmental and human isolates of Vibrio cholerae and Vibrio parahaemolyticus produce a Shigella dysenteriae 1 (Shiga)-like cytotoxin. Lancet 1984; 1: 77-78.

19. Datta-Roy K, Banerjee K, De SP, Ghose AC. Comparative study of expression of hemagglutinins, hemolysins, and enterotoxins by clinical and environmental isolates of non-O1 Vibrio cholerae in relation to their enteropathogenicity. Appl Environ Microbiol 1986; 52: 875-879.

20. Hanne LF, Finkelstein RA. Characterization and distribution of the hemagglutinins produced by Vibrio cholerae. Infect Immun 1982; 36: 209-214.

21. Sanyal SC, Alam K, Neogi PKB, Huq MI, Al-Mahmud KA. A new cholera toxin. Lancet 1983; 1: 1337.

22. Sanyal SC, Neogy PKB, Alam K, Huq MI, Al-Mahmud KA. A new enterotoxin produced by $V$. cholerae O1. J Diarrhoeal Dis Res 1984; 2: 3-12.

23. Saha S, Sanyal SC. Cholera toxin gene-positive Vibrio cholerae $\mathrm{O} 1 \mathrm{Ogawa}$ and Inaba strains produce the new cholera toxin. FEMS Microbiol Lett 1988; 50: 113-116.

24. Saha S, Sanyal SC. Neutralisation of the new cholera toxin by antiserum against crude enterotoxin of cholera toxin genepositive Vibrio cholerae $\mathrm{O} 1$ in rabbit ileal loop model. Indian $J$ Med Res 1989; 89: 117-120.
25. Saha S, Sanyal SC. Immunobiological relationships among new cholera toxins produced by CT gene-negative strains of Vibrio cholerae O1. J Med Microbiol 1989; 28: 33-37.

26. Saha S, Sanyal SC. Immunobiological relationships of the enterotoxins produced by cholera toxin gene-positive $\left(\mathrm{CT}^{+}\right)$ and negative $\left(\mathrm{CT}^{-}\right)$strains of Vibrio cholerae $\mathrm{O} 1 . J \mathrm{Med}$ Microbiol 1990; 32: 33-37.

27. World Health Organization. Manual for laboratory investigations of acute enteric infections. Geneva, WHO CDD/83.3. 1987

28. Iwanaga $\mathrm{M}$, Yamamoto $\mathrm{K}$, Higa $\mathrm{N}$, Ichinose $\mathrm{Y}$, Nakasone $\mathrm{N}$, Tanabe M. Culture conditions for stimulating cholera toxin production by Vibrio cholerae $\mathrm{O} 1 \mathrm{El}$ Tor. Microbiol Immunol 1986; 30: 1075-1083.

29. Annapurna E, Sanyal SC. Enterotoxicity of Aeromonas hydrophila. J Med Microbiol 1977; 10: 317-323.

30. De SN, Chatterje DN. An experimental study of the mechanism of action of Vibrio cholerae on the intestinal mucous membrane. J Pathol Bacteriol 1953; 66: 559-562.

31. Dubey RS, Sanyal SC. Enterotoxicity of Aeromonas hydrophila: skin responses and in vivo neutralisation. Zentralbl Bakteriol Orig A 1978; 242: 487-499.

32. Singh SJ, Sanyal SC. Enterotoxicity of the so-called NAG vibrios. Ann Soc Belg Med Trop 1978; 58: 133-140.

33. Shanker P, Agarwal RK, Sanyal SC. Experimental studies on enteropathogenicity of Vibrio cholerae serotypes other than O1. Zentralbl Bakteriol Mikrobiol Hyg A 1982; 252: 514-524.

34. Annapurna E, Sanyal SC. Studies on enteropathogenicity of Aeromonas hydrophila: an experimental model. Indian J Prev Soc Med 1975; 6: 234-237.

35. Sanyal SC, Singh SJ, Sen PC. Enteropathogenicity of Aeromonas hydrophila and Plesiomonas shigelloides. J Med Microbiol 1975; 8: 195-198.

36. Singh DV, Sanyal SC. Haemolysin and enterotoxin production by Aeromonas caviae isolated from diarrhoeal patients, fish and environment. $J$ Diarrhoeal Dis Res 1992; 10: 16-20.

37. Singh DV, Sanyal SC. Enterotoxicity of clinical and environmental isolates of Aeromonas spp. $J$ Med Microbiol 1992; 36: 269-272.

38. Sanyal SC, Saraswathi B, Sharma P. Enteropathogenicity of Plesiomonas shigelloides. J Med Microbiol 1980; 13: 401-409.

39. Sanyal SC, Agarwal RK, Annapurna E, Lee JV. Enterotoxicity of group F vibrios. Jpn J Med Sci Biol 1980; 33: 217-222.

40. Sanyal SC, Huq MI, Neogi PKB et al. Vibrio mimicus as an aetiological agent of diarrhoea and its pathogenesis. Indian $J$ Med Microbiol 1983; 1: 1-12.

41. Raychaudhury A, Agarwal RK, Sanyal SC. Enteropathogenicity of Klebsiella pneumoniae strains isolated from stools of diarrhoeal patients and other clinical specimens: an experimental study. Zentralbl Bakteriol Mikrobiol Hyg A 1984; 258: 94-103.

42. Dubey RS, Lindblad M, Holmgren J. Purification of El Tor cholera enterotoxins and comparisons with classical toxin. $J$ Gen Micriobiol 1990; 136: 1839-1847.

43. Tamplin ML, Ahmed MK, Jalali R, Colwell RR. Variation in epitopes of the B subunit of El Tor and classical biotype Vibrio cholerae Ol cholera toxin. J Gen Microbiol 1989; 135 $1195-1200$ 